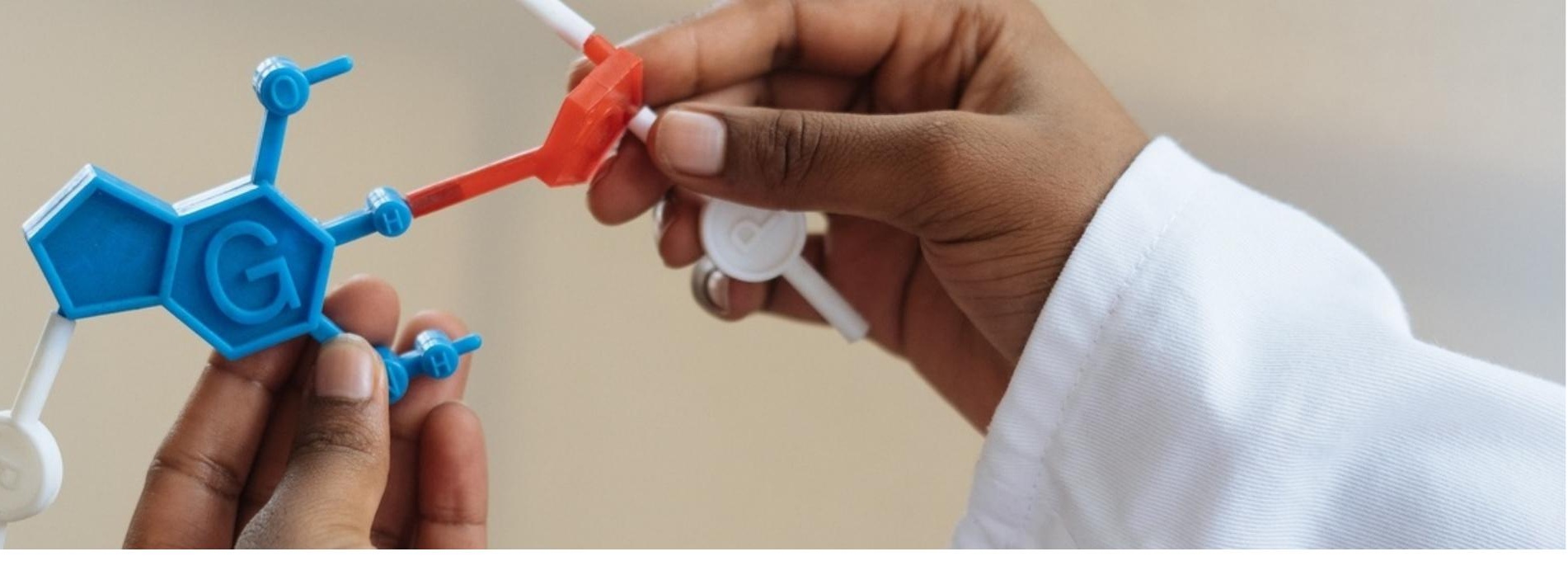

\title{
Artificial Intelligence and mathematical Models in Research and applied Oncology and Hematology
}

\author{
Authors: Roland Mertelsmann \\ Submitted: 29. January 2021 \\ Published: $\quad$ 8. February 2021 \\ Volume: 8 \\ Issue: 1 \\ Affiliation: BioThera Institut GmbH, Freiburg, Germany \\ Languages: \\ Keywords: \\ Categories: \\ English \\ Cancer, Artificial Intelligence, Research, Oncology, Hematology \\ Artificial Intelligence, Modeling and Simulation, Humanities, Social \\ Sciences and Law \\ DOI: $\quad$ 10.17160/josha.8.1.736
}

\section{Abstract:}

Since there is an infinite number of mutations that cause cancer, and even more possible combinations than there are molecules in the universe (Califano, Science 2020), the question arises whether the conventional approach of cancer research in the laboratory ("trial-error-trial") ) is realistic to recognize the important cancer-related patterns. Artificial intelligence has already shown that it can learn to recognize patterns in complex data structures such as face recognition. This approach is already being used in the discovery of new antibiotics and should also drive the discovery of novel cancer drugs. The extent to which these findings will find their way into clinical cancer therapy remains to be seen. The next step is the validation of these theoretical findings "in vitro" on cancer cells, followed by animal experiments and clinical studies. The results so far are optimistic, but the path to the clinic will still pose a lot of challenges for science.

\section{JOSHA Joumua os simmea Humanities and Arts}



und mathematische Modelle in der Forschung und angewandten Onkologie und Hämatologie 
www.af-institute.at

(C) 2020 AF Private Institute for Research and Ethical Use of Artificial Intelligence $\mathrm{GmbH}$.

Grünbergstraße 15, Stiege 2, Top 1.2a, A-1120 Vienna, Austria. All rights reserved. 


\section{Künstliche Intelligenz und mathematische Modelle in der Forschung und angewandten Onkologie und Hämatologie ${ }^{1}$}

\section{Prof. em. Dr. Drs. h.c. Roland Mertelsmann²}

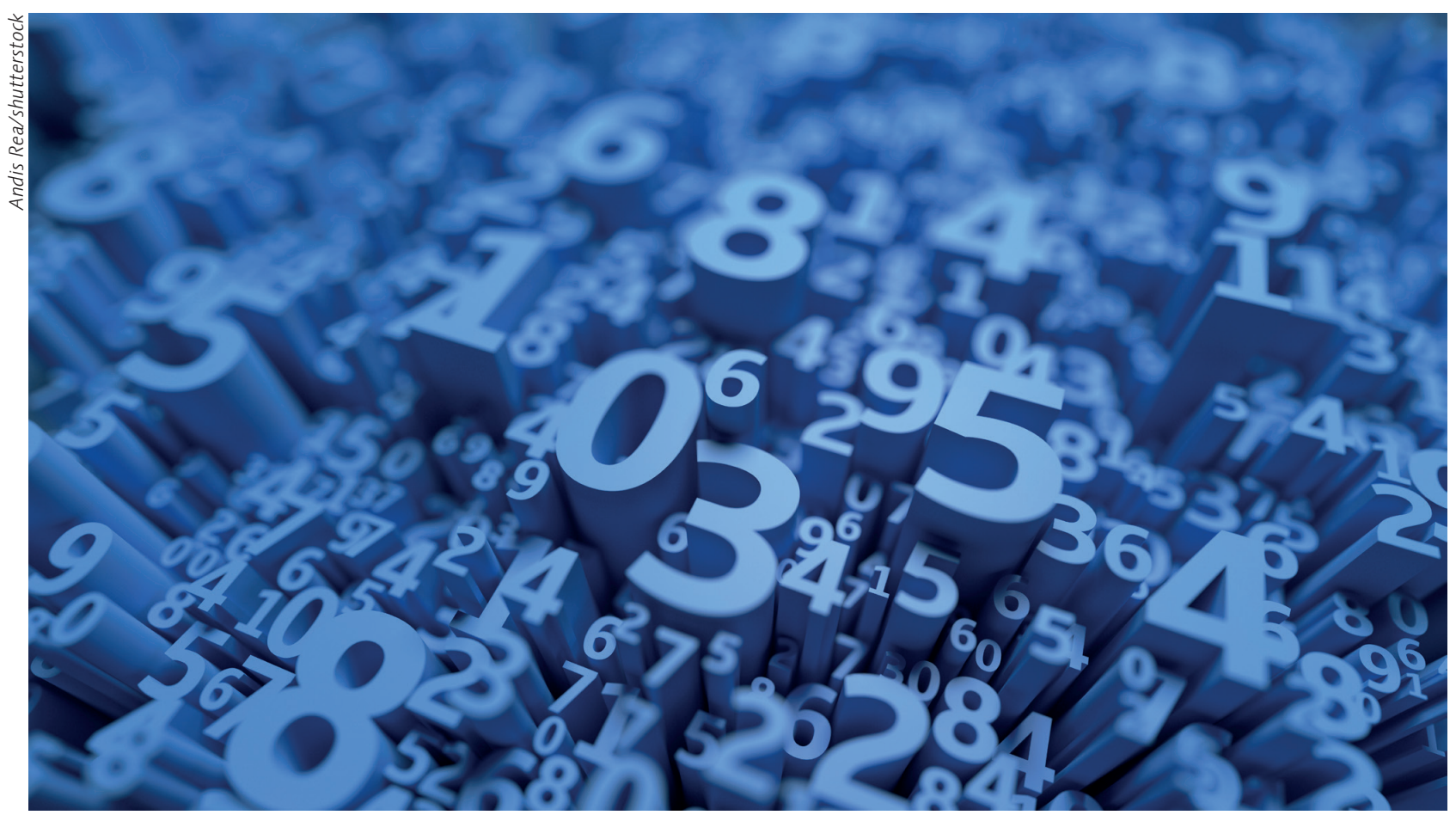

Inwieweit können Mathematik und Künstliche Intelligenz dazu beitragen, Krebstherapien zu verbessern? Dieses Paper geht dieser Frage nach und gibt einen kurzen Einblick in verblüffende Experimente und spannende neue Forschungsbereiche.

Professor Mertelsmann arbeitet an der Anwendung von KI in der translationalen Krebsmedizin zusammen mit einer multidisziplinären, internationalen Gruppe von Experten. Danksagung des Autors: Mein besonderer Dank gilt, pars pro toto, Dr. Sascha Lange, Jona Boeddinghaus, Professor Joschka Boedecker, Jonas Bermeitinger, Dennis Raith, Maximiliano Perez und "meinen" Studentinnen und Studenten.

1 Transkribierte und bearbeitete Zusammenfassung einer Keynote des Autors am 22.10.2020 beim Symposium „KI \& Krebs Erkenntnisgewinnung in der Krebsforschung durch Künstliche Intelligenz" im Haus der Industrie in Wien. Veranstalter war das AF Institute.

2 Roland Mertelsmann war viele Jahre international als Onkologe und Hämatologe tätig, zuletzt als Direktor der Abteilung für Medizin I, Onkologie, Hämatologie und Stammzelltransplantation am Uni-Klinikum Freiburg. Der emeritierte Professor wurde mehrfach ausgezeichnet und greift bei seinen Forschungen auf KI und mathematische Modelle zurück. 


\section{Automatisierte Röntgenbildauswertung}

\section{Auswertung vergleichbar zu der eines Panels aus drei praktizierenden Radiologen}

Auswertungszeit für 420 Bilder: $240 \mathrm{~min}$ (Radiologe) vs 1,5 min (Algorithmus)

(Rajpurkar et al., PLOS Medicine, 2018)
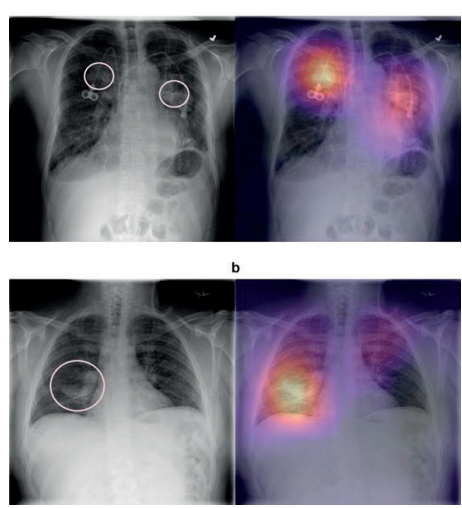

K rebs ist nach wie vor die zweithäufigste Todesursache in Ländern mit höherem Einkommen. 1970 wurden in den USA 625.000 neue Krebspatienten diagnostiziert mit einer Fünf-Jahres-Lebenserwartung von 49 Prozent.

Jetzt, 50 Jahre später, wo die Risikogruppe viel größer geworden ist, sind 1,8 Mio. neue Krebspatienten diagnostiziert worden bzw. werden noch diagnostiziert mit einer FünfJahres-Lebensrate von etwa 70 Prozent.

Es ist also eine Menge erreicht worden, aber diese Zahl heißt umgekehrt auch, dass noch 600.000 Krebspatienten in weniger als fünf Jahren versterben.

\section{Kann KI die Krebstherapie optimieren?}

Der Gedanke, vielleicht Mathematik in die Krebsforschung zu bringen, ist naheliegend. Mithilfe von Mathematik können wir Krebs besser verstehen. Das wiederum ermöglicht uns, neue therapeutische Konzepte zu entwickeln.

Ich berufe mich auf Immanuel Kant, der 1786 behauptete, "dass in jeder besonderen Naturlehre nur so viel eigentliche Wissenschaft angetroffen werden könne, als darin Mathematik anzutreffen ist."
Im Bereich der biomedizinischen Forschung gibt es in puncto Mathematik ganz sicher Nachholbedarf.

Deshalb stellt sich die Frage: Kann KI/AI - ich benutze die beiden Begriffe synonym die Krebstherapie optimieren, vielleicht sogar den Krebs besiegen?

Im Folgenden möchte ich auf diese Frage näher eingehen.

\section{Wo stehen wir mit $\mathrm{KI}$ in der klinischen Onkologie heute?}

In der Diagnostik findet man bereits Beispiele für die Anwendung von Künstlicher Intelligenz.

Zwei Beispiele möchte ich hervorheben, zunächst die Erkennung von Lungenmetastasen (Abb.1).

Hier wurde verglichen, was drei Radiologen auf 420 Röntgenbildern erkennen können und was ein KI-Algorithmus sieht, der selbst gelernt hat, Metastasen zu erkennen.

Das Ergebnis dieses Vergleichs zwischen Mensch und Maschine: Die Qualität der Resultate von KI-Agenten und Onkologen unterschied sich in diesem Experiment nicht wesentlich. Aber: Radiologen haben für die Analyse der Bilder vier Stunden gebraucht, der Algorithmus war in 90 Sekunden fertig. 


\title{
Hautkrebserkennung
}

\section{Auswertung von Muttermalen vergleichbar einer Gruppe von 21 praktizierenden Dermatologen}

\author{
Ausführung auf dem Smartphone möglich
}

Ca. 130.000 Trainingsbilder, Einteilung in über 750 Klassen

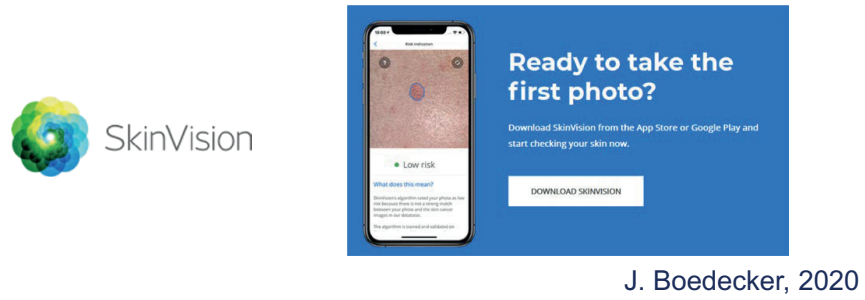

Abbildung 2

Nehmen wir dies als erstes Zwischenfazit: Ja, KI kann Aufgaben durchführen, die auch Menschen machen können, aber sehr, sehr viel schneller.

Ein zweites Beispiel ist die Hautkrebserkennung (Abb. 2): Auch hier hat man sich die Frage gestellt, inwieweit Hautkrebs von Dermatologen bzw. Algorithmen erkannt wird.

Die Maschine wurde mit über 130.000 Bildern von Muttermalen trainiert, eine Einteilung in über 750 Klassen wurde vorgenommen. Das Ergebnis: Der KI-basierte Agent hat in dieser Untersuchung Hautkrebs ungefähr genauso gut erkennen können wie die Dermatologen der Vergleichsgruppe.

Dabei ist man noch einen Schritt weiter gegangen. Mittels App erstellt man ein Foto eines Muttermals und innerhalb weniger Minuten erkennt die Software, ob es krebsverdächtig ist oder nicht. Kosten: rund 7 EUR.

\section{Erst am Anfang und schon "Standard"}

Die beiden Beispiele (Röntgenbild, Hautkrebs) zeigen, welche Geschäftsmodelle möglich sind und wie solide Bilderkennung arbeiten kann. Die Technologie steht erst am Anfang und ist dennoch schon "Standard".
Ein weiteres interessantes Thema: Man misst multiple Informationen aus Blut, zum Teil auch genetische Daten. Das Interessante dabei: Während keiner dieser Datenpunkte allein Krebs erkennen konnte, kann in der Gesamtschau der Daten ein Muster erkannt werden, das mit hoher Wahrscheinlichkeit auf Krebs hindeutet. Das ist work in progress, hier laufen gerade die Untersuchungen.

In der Analytik wird KI eingesetzt, um Muster in klinischen Daten zu erkennen, etwa Risikopatienten. Denken wir dabei z. B. an COVID: Fast jede Woche gibt es eine neue Publikation über neue Kombinationen von Risikofaktoren.

\section{Strukturen erkennen}

Man kann damit genauso Strukturen in Krebszellen erkennen, die vielleicht alle Krebszellen gemeinsam haben und deshalb für Therapeutika gute Zielstrukturen sein können. Man kann auch umgekehrt relevante Strukturen in bekannten und wirksamen Medikamenten identifizieren.

Das ist dieser analytische Teil in der Forschung. Das Prinzip dabei (Abb. 3): Wir sammeln Big Data - viele, viele Daten - und mithilfe von KI erkennen wir Muster und letztendlich die relevanten Strukturen, aus denen 
man dann wieder entsprechende Schlüsse ziehen kann.

\section{Mehr als Datenanalyse}

Das wird so mit Medikamenten gemacht, aber auch mit Krebszellen. Künstliche Intelligenz kann aber mehr als Datenanalyse - KI kann auch die Realität messen.

Das möchte ich gerne anhand eines Experiments (Abb. 4) unserer Arbeitsgruppe der Freiburger Universität darlegen (Technische Fakultät, u.a. mit Sascha Lange, mit dem ich viele Jahre zusammengearbeitet habe). Aufgabe für den Agenten war es, ein Pendel senkrecht nach oben (!) zu stellen und dort zu halten, ohne dass es kippt.

Um das zu erlernen, konnte der Computer nur den Schlitten, an dem das Pendel hing, nach links und rechts fahren und das Pendel damit in Schwingung setzen.

Beim Versuch schwang also das Pendel mal in die eine und mal in die andere Richtung, mal stärker, mal schwächer, mal langsamer, mal schneller, mal drehte es sich. Das Verblüffende: Beim 236. Versuch schaffte es der Algorithmus, das Pendel tatsächlich in die Zielposition zu bringen. In diese Position kehrte es auch zurück, nachdem es von einer Versuchsperson leicht angestoßen wurde. Das zeigt sehr gut, zu welchen Leistungen $\mathrm{KI}-\mathrm{Al}$ gorithmen fähig sind.

\section{$\mathrm{KI}$ in der klinischen Praxis}

Ich habe oben ausgeführt, dass KI in der Diagnostik und in der Analytik eine große Rolle spielt. Jetzt stellt sich eben die Frage, ob wir die Möglichkeiten, die KI eröffnet, auch nutzen können, um direkt in real life irgendwann einmal in klinische Prozesse einzugreifen.

Wir haben uns die Aufgabe gestellt, dass $\mathrm{KI}$ nicht von Datenbanken lernen soll, sondern in real time von Daten, die regelmäßig im System ermittelt werden. Und dann stellt sich daran die Frage: Können wir ein solches System dazu nutzen, um das Wachstum von Krebszellen zu kontrollieren?

Wenn man sich die Vielzahl molekularer Veränderungen von Krebszellen ansieht, wie sie kürzlich auch in der Zeitschrift Science veröffentlicht worden sind, so sieht man unendlich viele Möglichkeiten von Mutationen. Was die Sache noch komplexer macht, ist, dass diese verschiedenen Mutationen natürlich miteinander interagieren. Dr. Andrea Ca-

\section{Big Data - Mustererkennung}

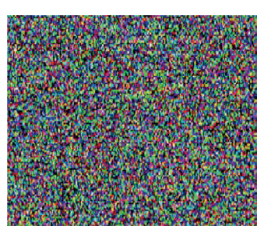

Big Data

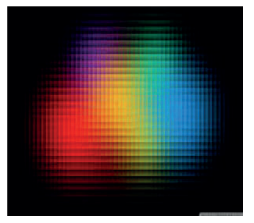

Big Picture 1
In verschiedenen Medikamenten ähnliche Strukturen erkennen - neue Medikamente synthetisieren mit diesen Eigenschaften

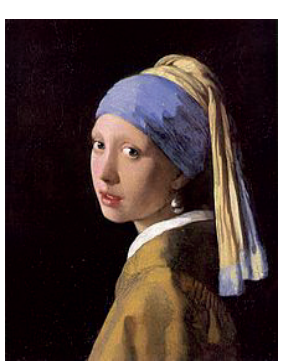

Big Picture 2 "Gestalt" 
lifano ${ }^{1}$ von der Columbia Universität fasst das so zusammen: Es gibt mehr mögliche Interaktionen an Krebsmutationen als Atome im Universum ("You have more potential combinations of cancerous mutations than atoms in the universe"). Da kann man sich leicht vorstellen, wie viele Experimente es brauchen würde, um das aufzuklären.

\section{Kann KI bei solchen Aufgaben helfen?}

Auch das Brettspiel Go ist eine Metapher dafür, dass $\mathrm{KI}$ einen Erfolg versprechenden Ansatz bilden kann. Bei Go gibt es unglaublich viele mögliche Positionen für die Figuren, nämlich rund $2,08 \times 10^{170}$. Die genaue Zahl hat 171 Stellen. Und auch hier lässt sich sagen, dass es wohl mehr mögliche Positionen gibt, als Atome im Universum ...

Die intellektuelle Herausforderung ist bei Go und Krebs ähnlich. KI kann die besten Schachspieler der Welt und die besten Gospieler der Welt besiegen. Bei Go gibt es mehr Positionsmöglichkeiten als Moleküle im Universum, bei Krebsmutationen gibt es ebenfalls mehr mögliche Kombinationen als Moleküle im Universum.

Damit stellt sich die Frage, ob KI nicht nur bei Go siegen hilft, sondern auch bei Krebs. Das Ziel mag hoch gegriffen sein, aber vielleicht unterstützt uns KI dabei, Therapien zu optimieren.

Mit meinen Freunden und Kollegen, darunter der Leiter des KI-Entwicklungsteams des AF Instituts, Jona Boeddinghaus, haben wir uns seit nunmehr zehn Jahren mit Computermodellen von Krebs und Krebs-Stoffwechsel beschäftigt.

Zunächst haben wir ein Krebsmodell entwickelt (Abb. 5), basierend auf den Hallmarks of Cancer von Douglas Hanahan und Robert Weinberg.

Wir haben darauf aufbauend das hier Gelernte in einem mathematischen Modell zur Leukämieentstehung und zum Wettbewerb zwischen leukämischen und gesunden Zellen umgesetzt. In der Folge haben wir einen spielerischen Ansatz entwickelt. Mittels eines

1 http://califano.c2b2.columbia.edu

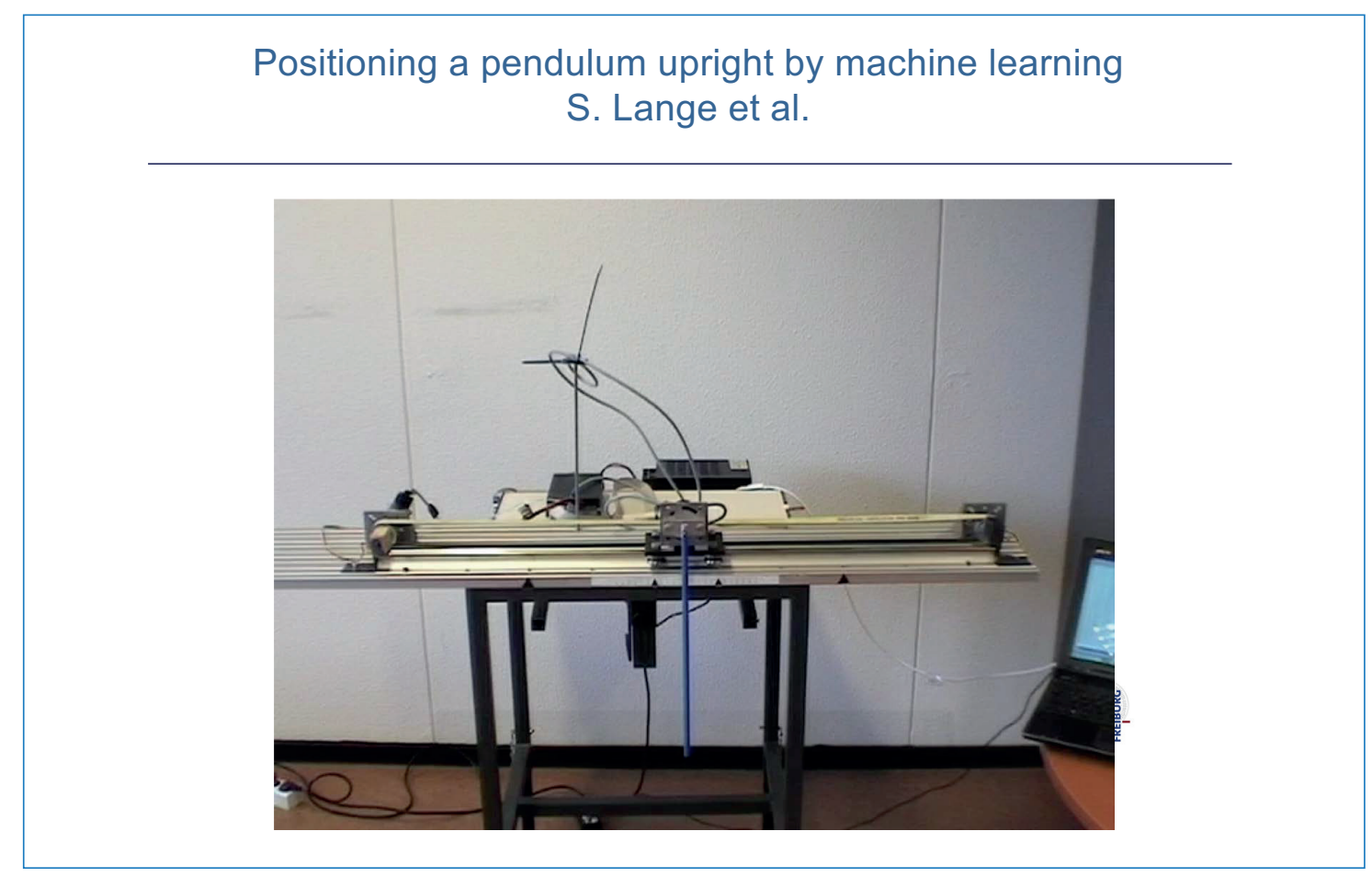

Abbildung 4 


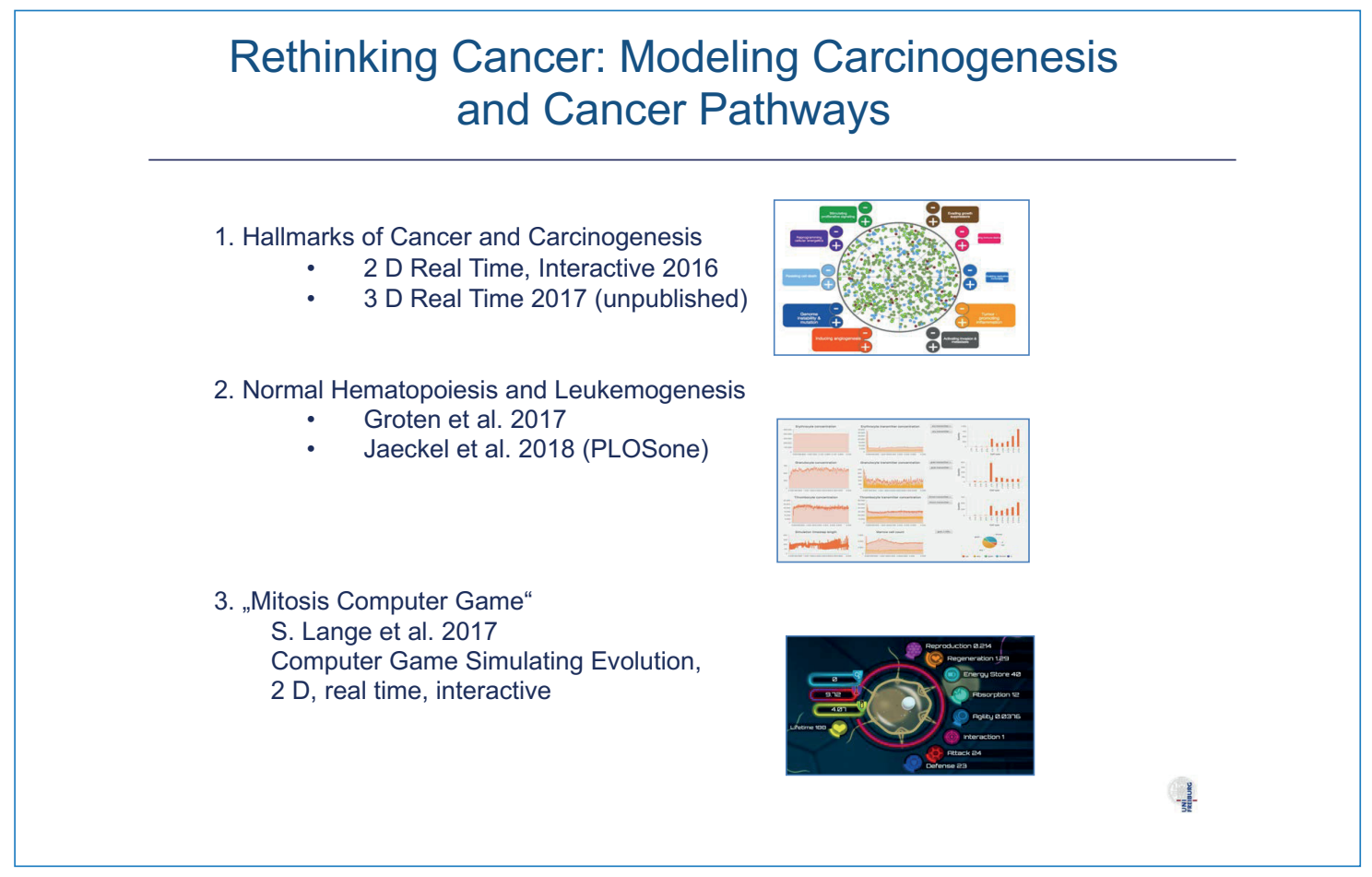

Abbildung 5

Computerspiels können wir zig Milliarden von Möglichkeiten ansehen.

\section{KI bei Behandlung von Krebszellen}

Und so stellt sich die nächste Frage: Kann KI auch direkt in die Behandlung von Krebszellen einwirken und uns helfen?

Im Augenblick forschen wir dazu mit Krebszellkulturen - die Zellen werden kontinuierlich analysiert (Diagnostik-Agent). Diese Zellen werden verschiedenen Krebsmedikamenten ausgesetzt, in unterschiedlichen Mengen, Dosierungen und Rhythmen.

Der KI-Agent Therapie steuert die Auswahl dieser Medikamente, der KI-Agent Diagnostik schaut sich dann das Ergebnis an. Anschließend adjustiert der KI-Agent Therapie die Therapie, um das gewünschte Ziel zu erreichen. Das Ziel könnte z. B. die folgende Optimierung sein: Gesunde Zellen wachsen, kranke Zellen sterben. Wir gehen davon aus, dass dieses System in der Lage sein sollte, eine Auswahl bezüglich der Medikamente, Dosierung und Kombination von Zellen zu treffen - oder im Modellfall mit Krebszellen aus der Kultur.

Wir haben uns in einem ersten Ansatz gefragt, wie wir diesen Gedankengang weiter simplifizieren und trotzdem einen Konzept- beweis entwickeln können. Dabei haben wir uns gesagt: Unser optisches Bild ist Farbe, ob wir in unseren Modellen mit Zellen oder vereinfacht nur Farbe arbeiten, ist konzeptionell gleichgültig.

Dann die nächste Frage: Kann ein Agent, der Diagnostik oder auch Therapie steuert, die Farben so mischen, dass ein gewünschtes Ergebnis herauskommt?

Das Ganze wird durch ein RGB-Mikroskop mit einer entsprechenden Farbkamera observiert. Kann dabei ein gewünschtes optisches Ergebnis erzielt werden, ohne dieses zu programmieren? Der Agent selbst kann entscheiden, welche Farben oder Geschwindigkeit er wählt. Und das Ziel des Agenten ist es, ein vorher definiertes Farbergebnis zu erzielen, nicht durch Programmierung, sondern indem er es selbst lernt. Und das schafft unser Agent inzwischen in zehn Stunden.

In unserem Experiment fingen wir in der Mischkammer mit Farbe Weiß an und erreichen schnell eine Zielfarbe.

Die Farben werden laufend gemessen Rot, Grün, Blau - und nach mehreren Schritten erreicht er den gewünschten Farbton. Das macht Künstliche Intelligenz - nachdem sie es gelernt hat - mittlerweile in weniger als zehn oder 20 Minuten. 


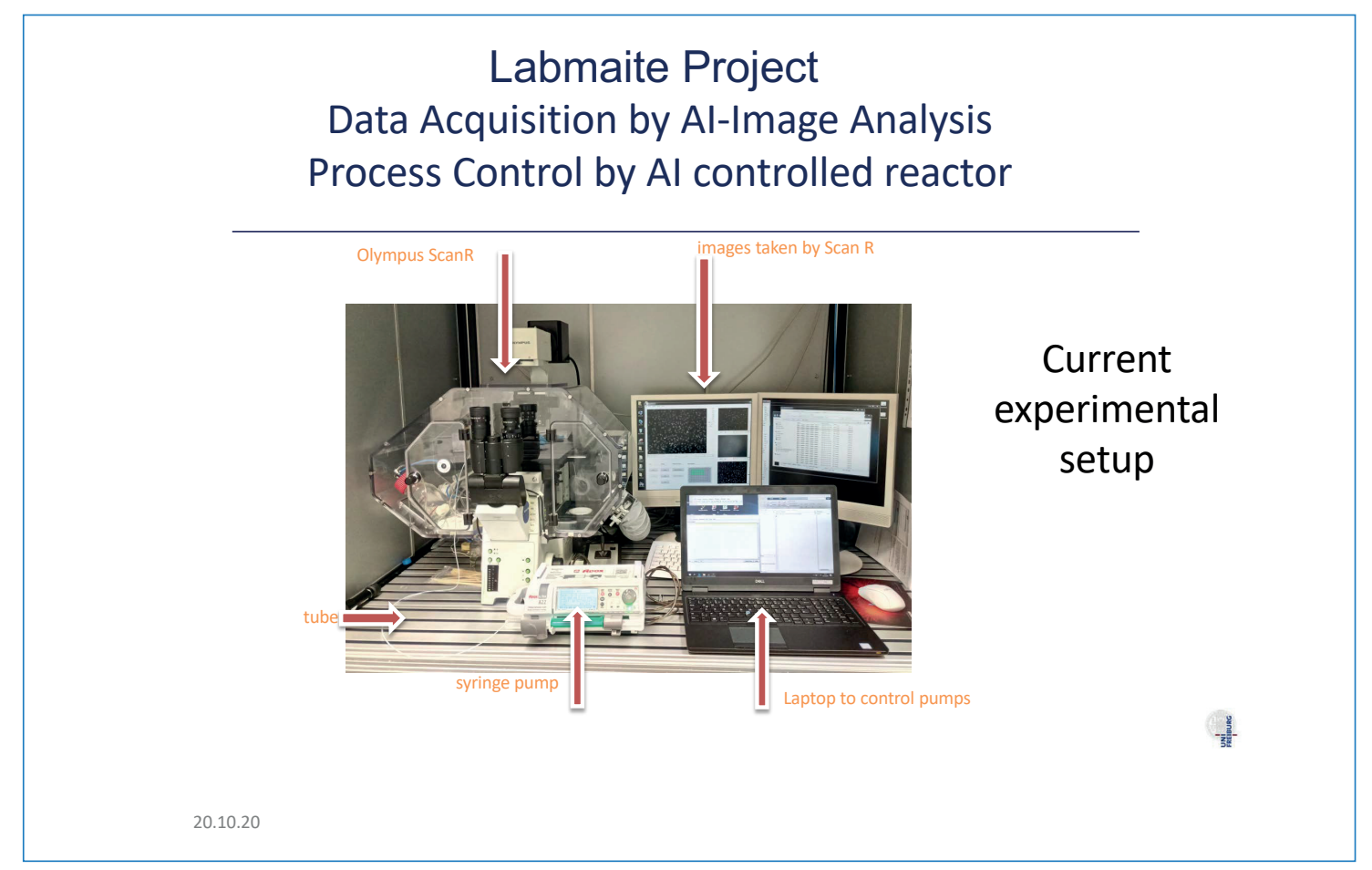

Abbildung 6

Das beweist, dass ein Agent auf KI basierend ein gewünschtes optisches Ergebnis in einem überschaubaren Zeitraum erreichen kann. Darauf basierend sind wir jetzt dabei, das Ganze mit Zellen zu machen.

Abb. 6 gibt Einblick in den Versuchsaufbau: Das Mikroskop erfasst die Bilder, die dann analysiert werden; die Information geht an den Agenten, der die Pumpen steuert und die Pumpen gehen wieder zurück in die Kamera. Das System funktioniert, was gar nicht einfach war: Es hat etwa 1,5 Jahre gedauert, bis alles zuverlässig lief.

\section{Zielvorgabe an die $\mathrm{KI}$}

Eine andere Versuchsreihe: Wir haben Kulturen von Brustkrebszellen durch Einsatz eines Chemotherapeutikums abgetötet. Zellen, die absterben, werden in der Visualisierung rot eingefärbt.

Das Ganze haben wir über einen Zeitraum von acht Stunden erfasst. In unseren Experimenten haben wir rund zehn Prozent der Zellen abgetötet.

In künftigen Experimenten werden wir dem Agenten vorgeben, dass wir als Ziel 30 Prozent der Zellen abtöten wollen. Der Agent kann dann aus vier Kanälen die richtige Medikamentenkombination wählen.

\section{Was haben wir bisher erreicht?}

Wir haben die Kultursysteme etabliert, die es erlauben, Langzeitkultur aufzubauen, also die Zellen über Wochen und gegebenenfalls auch Monate wachsen zu lassen - in Kulturen, in verschiedenen Systemen, verschiedenen Krebszelllinien, auch menschliche T-Lymphozyten, die auch in der Therapie eine Rolle spielen. Und wir befassen uns mit Hefe als Modellorganismus.

Der Agent kann in real life mit vier Variablen arbeiten, das Computermodell schafft das mit mehr als zehn Variablen, also mit mehr als zehn Chemotherapiesubstanzen.

Wir haben gezeigt, dass es mit Farbveränderung geht.

Im Augenblick laufen die Experimente, auch den $\mathrm{pH}$-Wert mithilfe einer optischen Analyse einzustellen. Auch die Untersuchung mit den Krebszellen läuft im Augenblick.

\section{Ausblick}

Ich bin sicher, dass KI die Krebstherapie optimieren wird. Es wird noch eine Weile dauern, aber es ist machbar. Bisher haben alle unsere Experimente geklappt, auch wenn es län- 


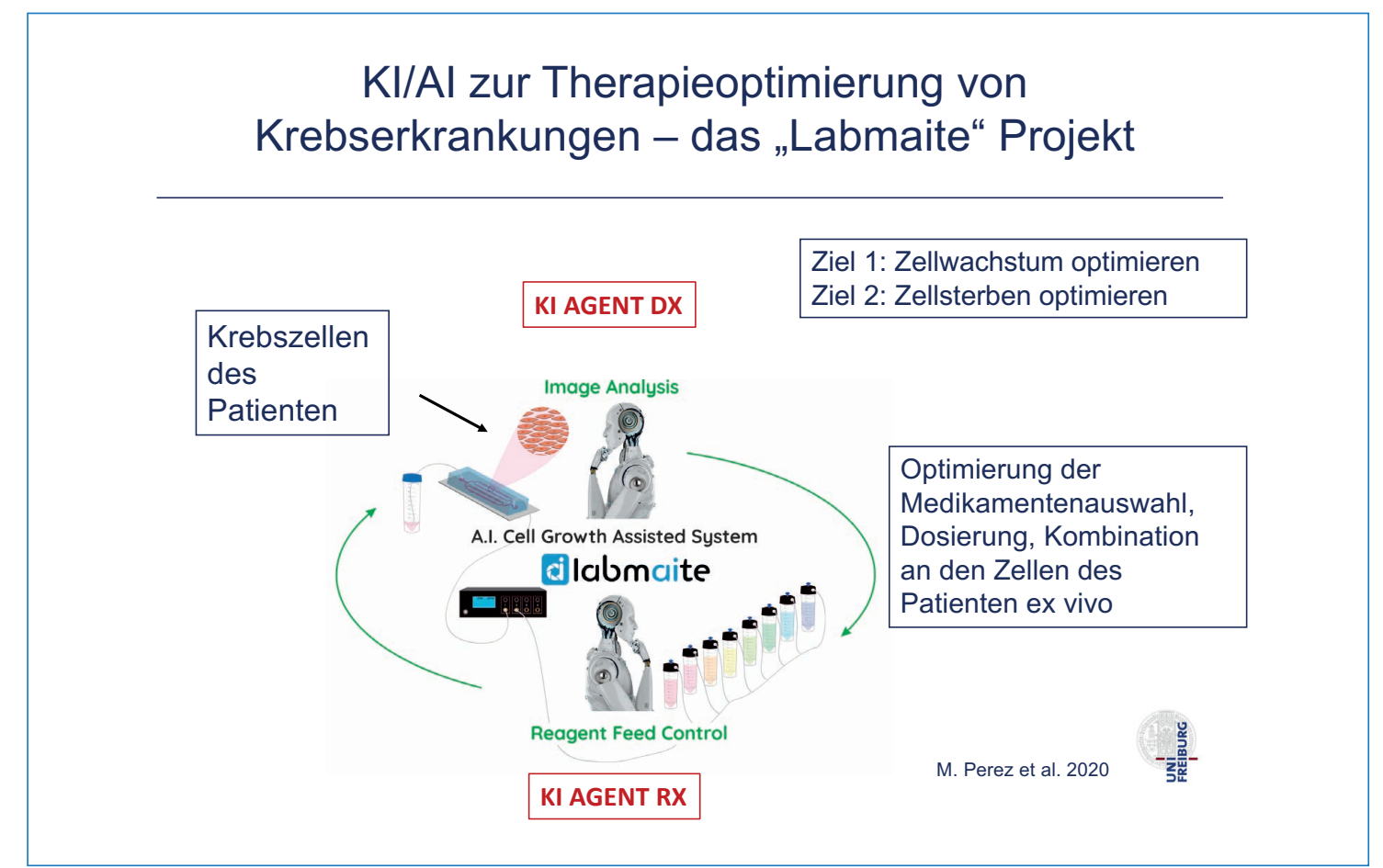

Abbildung 7

ger gedauert hat, als wir gehofft hatten. Wir werden den Krebs damit sicher nicht besiegen können. Aber ein anderes Ziel werden wir mit hoher Wahrscheinlichkeit erreichen, nämlich einen Beitrag dazu zu leisten, das Schicksal unserer Krebspatienten zu verbessern. 

AF INSTITUTE

Working Paper 